Check for updates

Cite this: Soft Matter, 2018,

14,5950

Received 28th March 2018

Accepted 21st May 2018

DOI: $10.1039 / \mathrm{c} 8 \mathrm{sm} 00651 \mathrm{~b}$

rsc.li/soft-matter-journal

\section{Construction of supramolecular hydrogels using photo-generated nitric oxide radicals $\dagger$}

\author{
Matthew Mulvee, (D) ab Natasa Vasiljevic, (D) bc Stephen Mann (D) a and \\ Avinash J. Patil (iD *a
}

\begin{abstract}
Photo-generated nitric oxide radicals (NO*) derived from sodium nitroprusside dihydrate (SNP) are employed for the construction of supramolecular hydrogels based on an amino acid derivative precursor, $\mathrm{N}$-fluorenylmethyloxycarbonyl tyrosine phosphate (FYP), which through dephosphorylation produces the gelator, $\mathrm{N}$-fluorenylmethyloxycarbonyl tyrosine (FY). Self-assembly of the amphiphilic gelator yields high-aspect ratio nanofilaments that entangle to form self-supporting, viscoelastic hydrogels. The presence of photolyzed SNP yields periodically twisted nanofilaments with opposite chirality to filaments formed through conventional hydrogelation routes.
\end{abstract}

Supramolecular hydrogels are formed through the self-assembly of low molecular weight gelators into high aspect ratio nanofilaments, which then entangle or branch at defect points to form a threedimensional solid-like network. Such highly entangled networks of nanofilaments act as a scaffold to immobilise water molecules through surface tension and capillary forces to form a viscoelastic hydrogel. ${ }^{1-5}$ Typically, this process requires a stimulus that causes a change in environment for the gelator, promoting self-assembly into extended and entangled nanofilaments. This can be a physical or chemical perturbation to the hydrogelator solubility. Most often, a gelator is dissolved at an elevated temperature, such that cooling results in supersaturation and subsequent hydrogelation. ${ }^{6-8}$ Enzymatic and biomimetic approaches have also been employed to form supramolecular hydrogels. For example, alkaline phosphatase $(\mathrm{ALP})^{9}$ and cerium oxide nanoparticles ${ }^{10}$ have been used to trigger gelation of FMOC-L-tyrosine-phosphate (FYP) via dephosphorylation of the tyrosine residue. Consequently, the ionic group $\left(-\mathrm{PO}_{3}{ }^{2-}\right)$ is converted into a neutral group $(-\mathrm{OH})$, reducing the electrostatic

\footnotetext{
${ }^{a}$ Centre for Organized Matter Chemistry and Centre for Protocell Research,

School of Chemistry, University of Bristol, Bristol, BS8 1TS, UK.

E-mail: Avinash.patil@bristol.ac.uk

${ }^{b}$ Bristol Centre for Functional Nanomaterials, University of Bristol, Tyndall Avenue, Bristol, BS8 1TL, UK

${ }^{c}$ School of Physics, University of Bristol, Bristol, BS8 1TS, UK

$\dagger$ Electronic supplementary information (ESI) available: Experimental details, SNP structure, ${ }^{13}$ C NMR, TEM, EDX, and CD data. See DOI: 10.1039/c8sm00651b
}

repulsion between molecules and their hydrophilicity. This promotes self-assembly through non-covalent interactions (hydrophobic, $\pi-\pi$ stacking, van der Waals, electrostatic), into one-dimensional protofilaments, which laterally associate to produce nanofibers. Interactions between the planar FMOC groups along with H-bonding between the chiral FMOC-tyrosine (FY) groups are thought to be key driving forces for the assembly of the gelator molecules into filaments with $\beta$-sheet-like structure and interlocked aromatic stacking. ${ }^{9,11-13}$ At a critical density, the entangled network of nanofibers is capable of entrapping water molecules to produce a self-supported hydrogel.

Gelator assembly is thought to be kinetically dependent, affecting nucleation processes and growth rates of the nanofilaments. Additionally, supramolecular assembly is a delicate balance of repulsive forces (e.g. electrostatics) and attractive forces (e.g. hydrophobic interactions, H-bonding etc.). Therefore, the environment in which this assembly occurs can modulate these interactions through conditions such as varying ionic strength, temperature etc. to affect the structures formed. As a result, various stimuli and experimental conditions can have dramatic effects on the mechanical properties of the hydrogel as well as the gelation time, filament and network structure, and optical properties. ${ }^{14-21}$ As a consequence, investigations into novel stimuli and their effect on hydrogel properties are of immediate interest. In this regard, despite the extensive use of free radicals to crosslink polymeric hydrogels, ${ }^{22}$ the utilization of radicals for the construction of supramolecular hydrogels remains unexplored.

Herein, we show that UV radiation-induced photolysis of sodium nitroprusside dihydrate (SNP) generates nitric oxide radicals, which can cleave the phosphoester of FYP to form FY molecules that subsequently self-associate to produce a selfsupported supramolecular hydrogel (Fig. 1 and Fig. S1, ESI $\dagger$ ). Importantly, this could be done at room temperature and by simply irradiating samples to UV light for approximately 30 minutes. Significantly, we show that the radical-induced dephosphorylation and presence of the photolyzed product of SNP, pentacyanoferrate(III) hydrate, has dramatic effects on 
(a)

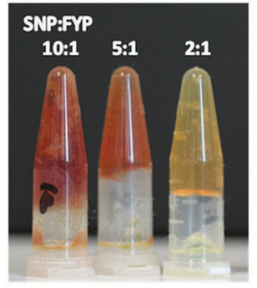

(b)

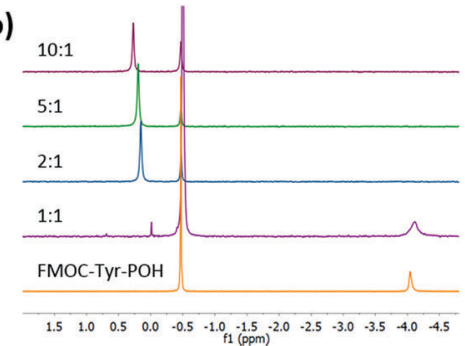

Fig. 1 (a) Photograph showing self-supported supramolecular hydrogels prepared from various SNP: FYP molar ratios; (b) ${ }^{31}$ P NMR spectra of corresponding hydrogel samples showing nitric oxide-mediated dephosphorylation of FYP. Sample prepared at 1:1 showed only partial dephosphorylation of FYP and therefore failed to produce a self-supported hydrogel.

non-covalent (i.e., H-bonding, $\pi-\pi$ ) interactions between the FY gelator molecules and thereby on their self-assembly. Consequently, self-assembled nanofilaments show left-handed supramolecular chirality, rather than the right-handed chirality observed in hydrogels produced via conventional alkaline phosphatase-mediated dephosphorylation of FYP (ALP-FY).

Nitric oxide radical-induced hydrogels were produced by mixing an aqueous solution of SNP and buffered solution of FYP at molar ratios (SNP:FYP) $10: 1,5: 1,2: 1$ and $1: 1$, while maintaining the concentration of FYP at $50 \mathrm{mM}$. These mixtures were irradiated with a UV lamp $(\lambda=254 \mathrm{~nm})$ for 30 minutes. Interestingly, except for the sample with molar ratio $1: 1$, all other samples passed the inversion test indicating the formation of selfsupported hydrogels (Fig. 1a). The orange coloured appearance of the hydrogels was due to the iron of the SNP. Control experiments with a mixture of SNP and FYP kept in the dark did not produce a hydrogel. Significantly, SNP solutions were stable in the dark and did not form any products, indicating that photo-irradiation was necessary to trigger supramolecular gelation. ${ }^{23-25}$ Furthermore, no gels were formed when either SNP or FYP were absent from the reaction mixture (Fig. S2, ESI $\dagger$ ).

The ${ }^{31} \mathrm{P}$ NMR spectrum of FYP exhibited a characteristic resonance at $-4.05 \mathrm{ppm}$ corresponding to the phosphate ester functionality attached to an aryl group, which disappeared in the hydrogel samples. Concomitantly, a new peak at $0.20 \mathrm{ppm}$ observed in the gel samples was attributed to inorganic phosphate (Fig. 1b). This was consistent with ${ }^{13} \mathrm{C}$ NMR spectra (Fig. S3, ESI†). The ${ }^{13} \mathrm{C}$ spectrum of FYP displayed a resonance at $150.57 \mathrm{ppm}$ associated with an aryl $\mathrm{C}-\mathrm{O}-\mathrm{P}(\mathrm{O})(\mathrm{OH})_{2}$, which was found to be absent in all the hydrogel samples. Instead, it was replaced with a peak at $152.86 \mathrm{ppm}$, corresponding to an aryl $\mathrm{C}-\mathrm{OH} .^{10,26}$ These observations were consistent with earlier reports in which SNP has been employed for the dephosphorylation of small dye molecules such as nitrophenyl phosphate, ${ }^{23,27,28}$ and indicates that nitric oxide radicals generated via photolysis of SNP are able to cleave the phosphate ester bond allowing the dephosphorylated product to yield SNP-FY supramolecular hydrogels.

Cryo-TEM analysis revealed that the SNP-FY gels consisted of thinly twisted nanofilaments that were $2-4 \mathrm{~nm}$ in diameter and several micrometres in length (Fig. 2a). The helical pitch of the nanofilament was ca. $130 \mathrm{~nm}$ (Fig. S4, ESI†). Similar observations

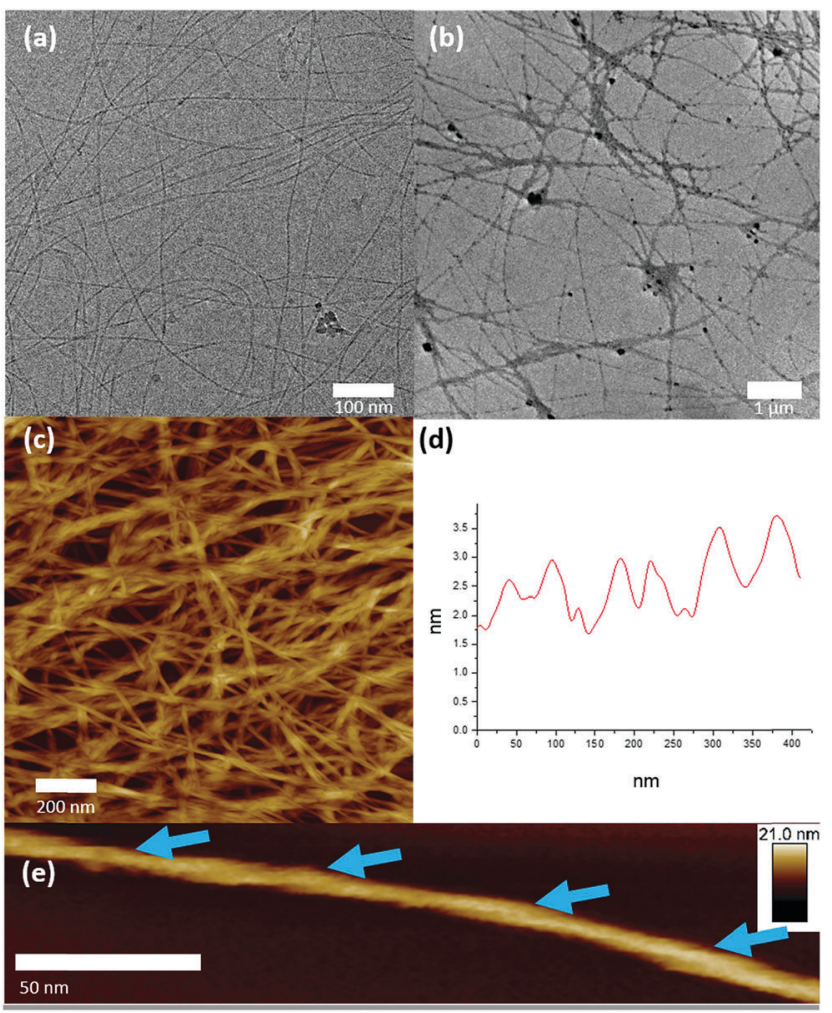

Fig. 2 (a) Cryo-TEM image of SNP-FY hydrogel showing narrow and twisted filaments (scale bar $=100 \mathrm{~nm}$ ), (b) unstained TEM image of SNP-FY hydrogel nanofilaments, decorated with SNP salts (scale $=1 \mu \mathrm{m}$ ), (c) AFM data of SNP-FY hydrogel, drop cast on mica, (d) height profile of nanofilaments, and (e) height scan showing the twisting of nanofilaments (arrows indicate location of twisting).

were made for the uranyl acetate stained samples viewed under TEM (Fig. S5c, ESI $\dagger$ ). In comparison, FY gels produced by conventional enzymatic routes showed slightly thicker twisted nanofilaments with a width of $c a .14 \mathrm{~nm}$ and a helical pitch of $c a .220 \mathrm{~nm}$ (Fig. S5d, ESI $\dagger$ ). Furthermore, conventional TEM analysis of unstained SNP-FY gel samples revealed that the nanofilaments were decorated with electron-dense inorganic particles/salts (Fig. 2b) and the corresponding energy dispersive X-ray spectroscopy analysis showed the presence of iron confirming the association of the SNP precursor with the nanofilaments (Fig. S6, ESI†). AFM images of the hydrogels drop-casted onto freshly cleaved mica showed a highly entangled network of nanofilaments. (Fig. 2c) The height profile images obtained from AFM analysis showed that the nanofilaments were $6.93 \mathrm{~nm}( \pm 0.51)$ thick and larger bundles had a periodic twist of $c a .130 \mathrm{~nm}$ that was consistent with cryo-TEM analysis (Fig. 2d and Fig. S4b, ESI $\dagger$ ). The observed twist in the filaments was attributed to $\mathrm{H}$-bonding between the chiral gelator molecules, which imparts curvature into the growing one-dimensional filaments, suppressing the formation of twodimensional sheets. ${ }^{11,29,30}$

Significantly, the AFM images revealed the presence of lefthanded helical nanofilaments rather than conventional righthanded helical filaments observed in previous studies involving FY (Fig. 2d). ${ }^{9,10}$ This interesting observation suggested that the 

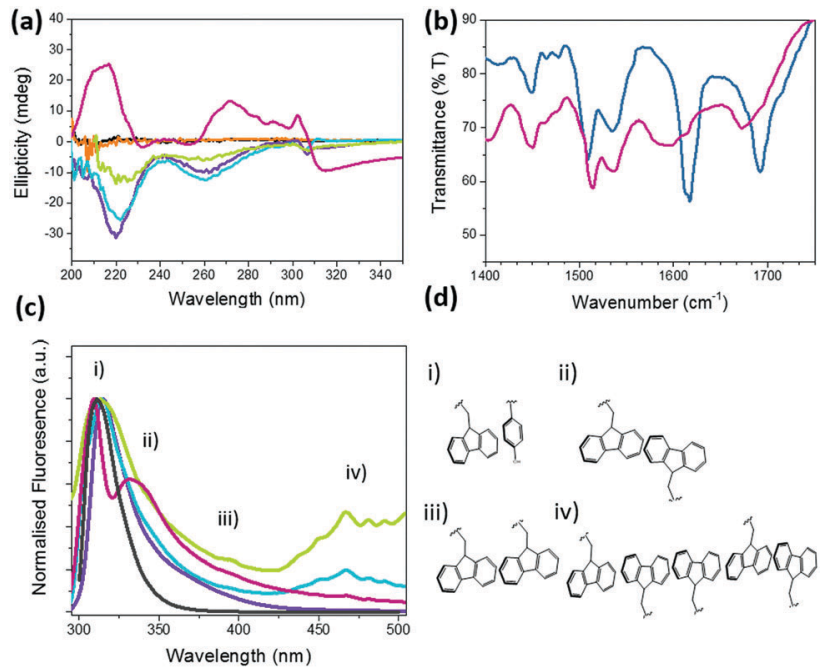

Fig. 3 (a) CD spectra of hydrogels prepared at varying molar ratios of FYP and SNP, and in the presence of alkaline phosphatase, (b) FT-IR spectra of SNP-FY hydrogel and gel obtained through enzymatic route, (c) emission spectra of hydrogels prepared at varying molar ratios, and (d) different aromatic packing arrangements of the gelator molecules. SNP: FYP ratios of $1: 1$ (purple), $2: 1$ (blue), $5: 1$ (green) and $10: 1$ (red), as well as FYP solution (black), SNP solution (orange) and ALP-FY gel (magenta) are displayed.

chiral packing of the radical-mediated dephosphorylated hydrogelator molecules was significantly altered. This was confirmed by circular dichroism (CD) spectroscopy. A CD spectrum of the control ALP-FY hydrogel showed characteristic positive signature peaks at 200-230 ( $\mathrm{n}-\pi^{*}$ transitions) and $270-310 \mathrm{~nm}\left(\pi-\pi^{*}\right.$ transitions) that were attributed to offset stacking of the fluorenyl and phenyl moieties. $^{31,32}$ Remarkably, $\mathrm{NO}^{\bullet}$ radical-mediated gelation revealed similar peaks but with opposite (negative) ellipticity (Fig. 3a). This was intriguing as the control hydrogel and SNP-FY hydrogels were formed using L-amino acid derivatives, and therefore the opposite ellipticity was due to opposite chirality inherent to the fibrillar assemblies. This was further supported by FTIR spectroscopy. FTIR spectra of SNP-FY gels exhibited a different H-bonding network compared to the ALP-FY gels. Both gels exhibited peaks between $1600-1700 \mathrm{~cm}^{-1}$ arising from the carbonyl H-bonding, indicative of a $\beta$-sheet like assembly (Fig. 3b). ${ }^{33,34}$ In the case of SNP-FY hydrogels, part of the region was masked by $\mathrm{O}-\mathrm{H}$ bending vibrations corresponding to water molecules weakly associated with SNP. ${ }^{35}$ However, the peak related to H-bonding originating from the carbamate group was significantly shifted by $20 \mathrm{~cm}^{-1}$ as well as a shift for the $\mathrm{N}-\mathrm{H}$ bending peak between $1500-1550 \mathrm{~cm}^{-1}$. These shifts to higher frequencies indicated a weakening of the intermolecular hydrogel bonding for the SNP-FY gels compared to the ALP-FY gels. ${ }^{36,37}$ The broad peak at $1565 \mathrm{~cm}^{-1}$ was only present for the ALP-FY gel and corresponded to the unprotonated carboxylic acid, ${ }^{18,34}$ arising from the higher $\mathrm{pH}$ of this gel and was not indicative of a different packing arrangement.

Anionic salts can polarise water molecules and thereby influence $\mathrm{H}$-bonding to peptides, and have direct interactions with the amide groups of gelators to affect their assembly. ${ }^{38,39}$ Thus, it is possible that either of these effects could directly alter the supramolecular packing of FY molecules in the SNP-FY hydrogels. This was further supported by control CD experiments which demonstrated that the supramolecular chirality of the hydrogels was influenced when SNP was present in the gelling solution but not used to trigger hydrogelation. For example, ALP-FY gel samples doped with SNP showed negative ellipticity indicating that SNP can interact with gelator molecules to alter the assembly process (Fig. S8a, ESI $\dagger$ ).

In light of these observations, H-bonding between SNP and FY may have an important role in controlling supramolecular chirality. For instance, the nitrogen of the cyanide ligand has been shown to be an effective H-bonding acceptor. ${ }^{40-42}$ Similarly, H-bonding donor interactions between hydroxyl and carbamate groups could offset carbamate-mediated H-bonding with the phenol group of FY. ${ }^{12}$ Additionally, the electron donating hydroxyl of the tyrosine residue polarises the $\pi$-system of the phenyl moiety. As a result, H-bonding between the pentacyanoferrate(III) hydrate and FY would lead to different interactions between the aromatic groups of FY compared with the enzyme-mediated hydrogelation system. Indeed shifts were observed relative to ALP-FY gel at $1402 \mathrm{~cm}^{-1}$ to $1413 \mathrm{~cm}^{-1}$ and $1446 \mathrm{~cm}^{-1}$ to $1438 \mathrm{~cm}^{-1}$, associated with the hydroxyl $(\mathrm{C}-\mathrm{O}-\mathrm{H})$ bending vibration of the tyrosine and ring vibrations, respectively. ${ }^{43,44}$ This clearly indicates changes to the $\mathrm{H}$-bonding for these groups. Furthermore, the electronic nature of the aromatic rings, which arises from the positively charged $\sigma$-framework located between two regions of negatively charged $\pi$ electron density on the face of the ring, is determined by substituents such as halogens that can tune the strength and geometry of the intermolecular aromatic interactions. ${ }^{45-48}$ In addition, the geometries for intermolecular $\pi-\pi$ interactions are partly determined by the quadrupole moment, and therefore any perturbation could conceivably encourage different stacking arrangements. ${ }^{45}$ This was consistent with the fluorimetry data (vide infra), and could be responsible for the formation of a different supramolecular structure.

Peptide-based fibrils formed through a $\beta$-sheet $\mathrm{H}$-bonding network are known to show variations in chirality due to assembly conditions, indicating that such a motif is sufficiently versatile to allow filaments of either handedness to form. ${ }^{49-51}$ Hence, as described above, a perturbed H-bonding arrangement could conceivably affect the curvature and consequently the supramolecular chirality of the hydrogel filaments. We therefore investigated the effect of temperature on the chirality of the nanofilaments. SNP-FY hydrogels heated at $80{ }^{\circ} \mathrm{C}$ and cooled to room temperature showed positive ellipticity (Fig. S8b, $\mathrm{ESI} \dagger$ ). This observation indicated that gelation in the presence of SNP leads to kinetically trapped structures, whilst heating and cooling cycles facilitate a more thermodynamically favoured state. $^{52-54}$

Beyond $\mathrm{H}$-bonding, another key driving force for the assembly of amphiphilic gelators is the $\pi-\pi$ stacking and hydrophobic interactions between the aromatic residues. ${ }^{9,11}$ The emission spectra for a FYP solution and SNP-FY and ALP-FY hydrogels are shown in Fig. 3c. The FYP solution exhibited an emission peak at $311 \mathrm{~nm}$, whereas the hydrogel samples showed a slight red-shift in their emission indicative of inefficient $\pi-\pi$ stacking, ${ }^{55,56}$ 
(a)

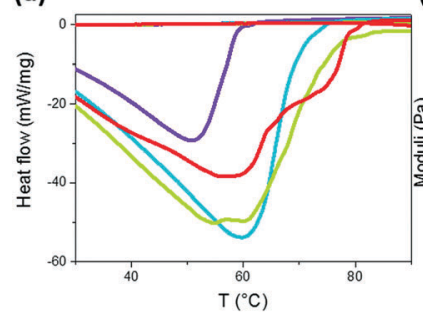

(b)

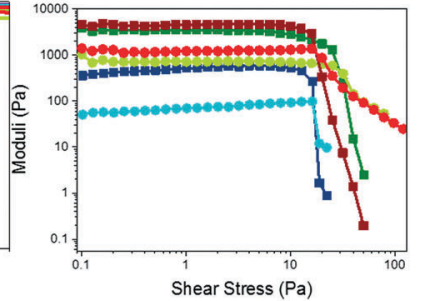

Fig. 4 (a) DSC thermogram of supramolecular hydrogels prepared from FYP $(50 \mathrm{mM})$ showing broad gel to sol transition temperatures that increase with increasing SNP: FYP molar ratios (b) oscillatory amplitude sweeps at a constant frequency $(1 \mathrm{~Hz})$. All gels exhibited a linear viscoelastic regime and the stiffness was affected by the SNP : FYP ratio. Symbols $(\boldsymbol{\square})$ and $(\bullet)$ represents storage/elastic moduli $G^{\prime}$ and viscous moduli $G^{\prime \prime}$, respectively. Samples prepared at SNP:FYP ratios of $1: 1$ (purple), 2:1 (blue), 5:1 (green) and 10:1 (red) are displayed.

which has been attributed to FMOC-phenyl stacking (Fig. 3d(i)). However, at higher wavelengths, the ALP-FY gel exhibits a peak at $331 \mathrm{~nm}$ associated with anti-parallel stacking of the FMOC residues (Fig. 3d(ii)), ${ }^{9}$ which is absent in the SNP-FY gels. In contrast, SNP-FY hydrogels showed a broad spectrum with a feature at $395 \mathrm{~nm}$ that was attributed to parallel stacking of FMOC residues (Fig. 3d(iii)). ${ }^{9}$ Significantly, with increasing SNP:FYP ratios there was an increase in intensity for the substantially red-shifted emission peaks, which were assigned to excimer formation as a result of extended and conjugated $\pi-\pi$ stacking interactions (Fig. $3 d(i v)){ }^{34,57}$ These observations suggested that the increased amounts of SNP present in solution facilitated extended aromatic stacking, which was in agreement with previous reports suggesting that hydrophobic interactions become dominant when salts are present during the assembly process. ${ }^{58,59}$

The structural and mechanical properties of the SNP-FY gels were investigated by DSC and rheology. DSC profiles showed a broad endothermic peak between 51 and $71{ }^{\circ} \mathrm{C}$ corresponding to gel to sol transitions (Fig. 4a). The melting temperatures of the SNP-FY hydrogels increased with increasing SNP:FYP ratios and were attributed to the organic-inorganic hybrid nature of the hydrogel samples. Viscosity measurements showed that at minimal shear, the viscosity of the hydrogels increased from 0.32 to $137.4 \mathrm{~Pa}$ s with increasing molar ratio of SNP:FYP, and decreased with increasing shear rates (shear thinning), which is typical of supramolecular hydrogels (Fig. S9, $\mathrm{ESI} \dagger)$. Oscillatory frequency sweeps showed a linear viscoelastic region (LVR) in which the storage (elastic) $G^{\prime}$ moduli were roughly an order of magnitude higher than the loss (viscous) $G^{\prime \prime}$ moduli for all stoichiometries, except 1:1 (Fig. S9 and S10, ESI $\dagger$ ). This observation was indicative of a solid-like network throughout the gel for all stoichiometries except at a $1: 1$ molar ratio. The viscous liquid-like properties of the SNP-FY (molar ratio $1: 1$ ) sample were attributed to an insufficient cross-linked network of nanofilaments required for hydrogelation. In this regard, the crossover points observed in the case of the oscillatory amplitude sweep could be assigned to structural deformation of the samples as they transformed from an elastic gel to a viscous fluid (Fig. 4b).

In summary, we show that photolysis of SNP produces nitric oxide radicals capable of dephosphorylation of FYP to yield supramolecular hydrogels. Significantly, AFM and spectroscopy techniques indicate that the photolyzed product of SNP (pentacyanoferrate(III) hydrate) has a significant effect on the self-assembly process of the dephosphorylated gelator molecules. In turn, this facilitates the formation of kinetically trapped, amyloid-like nanofilaments with left-handed helicity. Taken together, it is envisioned that nitric oxide radicals could be readily employed in the dephosphorylation of a wide range of important functional amino acid and peptide derivatives for the construction of supramolecular hydrogels with tuneable chirality.

We acknowledge the EPSRC (EP/G036780/1) and the Bristol Centre for Functional Nanomaterials for project funding. The authors thank Judith Mantel, Jennifer Coombs and the Wolfson Bioimaging Facility for their assistance with cryo-TEM, Dr Rob Harniman for assistance with AFM work, Jon Jones and Dr Sean Davis for assistance with TEM. The data supporting the findings of this study are available within the paper and its supplementary information files, and are available from the corresponding author upon reasonable request.

\section{Conflicts of interest}

There are no conflicts to declare.

\section{Notes and references}

1 L. A. Estroff and A. D. Hamilton, Chem. Rev., 2004, 104, 1201-1217.

2 R. G. Weiss and P. Terech, Molecular Gels, Springer, Netherlands, Dordrecht, 2006.

3 S. Ramachandran, P. Flynn, Y. Tseng and Y. B. Yu, Chem. Mater., 2005, 17, 6583-6588.

4 X. Du, J. Zhou, J. Shi and B. Xu, Chem. Rev., 2015, 115, 13165-13307.

5 Y. Zhang, H. Gu, Z. Yang and B. Xu, J. Am. Chem. Soc., 2003, 125, 13680-13681.

6 G. M. Peters and J. T. Davis, Chem. Soc. Rev., 2016, 45, 3188-3206.

7 N. M. Sangeetha and U. Maitra, Chem. Soc. Rev., 2005, 34, 821.

8 M. George and R. G. Weiss, Acc. Chem. Res., 2006, 39, 489-497.

9 Z. Yang, H. Gu, D. Fu, P. Gao, J. K. Lam and B. Xu, Adv. Mater., 2004, 16, 1440-1444.

10 A. J. Patil, R. K. Kumar, N. J. Barron and S. Mann, Chem. Commun., 2012, 48, 7934.

11 S. Fleming and R. V. Ulijn, Chem. Soc. Rev., 2014, 43, 8150-8177.

12 E. R. Draper, K. L. Morris, M. A. Little, J. Raeburn, C. Colquhoun, E. R. Cross, T. O. McDonald, L. C. Serpell and D. J. Adams, CrystEngComm, 2015, 17, 8047-8057.

13 X. Du, J. Zhou and B. Xu, Chem. - Asian J., 2014, 9, 1446-1472. 
14 M. A. Greenfield, J. R. Hoffman, M. Olvera de la Cruz and S. I. Stupp, Langmuir, 2010, 26, 3641-3647.

15 S. Roy, N. Javid, J. Sefcik, P. J. Halling and R. V. Ulijn, Langmuir, 2012, 28, 16664-16670.

16 W. Helen, P. de Leonardis, R. V. Ulijn, J. Gough and N. Tirelli, Soft Matter, 2011, 7, 1732-1740.

17 J. Raeburn, A. Zamith Cardoso and D. J. Adams, Chem. Soc. Rev., 2013, 42, 5143.

18 L. M. Carrick, A. Aggeli, N. Boden, J. Fisher, E. Ingham and T. A. Waigh, Tetrahedron, 2007, 63, 7457-7467.

19 B. Ding, Y. Li, M. Qin, Y. Ding, Y. Cao and W. Wang, Soft Matter, 2013, 9, 4672.

20 L. Chen, J. Raeburn, S. Sutton, D. G. Spiller, J. Williams, J. S. Sharp, P. C. Griffiths, R. K. Heenan, S. M. King, A. Paul, S. Furzeland, D. Atkins and D. J. Adams, Soft Matter, 2011, 7, 9721.

21 J. M. Poolman, J. Boekhoven, A. Besselink, A. G. L. Olive, J. H. Van Esch, R. Eelkema, J. H. van Esch and R. Eelkema, Nat. Protoc., 2014, 9, 977-988.

22 W. E. Hennink and C. F. van Nostrum, Adv. Drug Delivery Rev., 2012, 64, 223-236.

23 F. Tafesse and M. Enemchukwu, Nitric oxide, 2008, 18, 274-278.

24 A. R. Butler, F. W. Flitney and D. L. H. Williams, Trends Pharmacol. Sci., 1995, 16, 18-22.

25 A. C. Van Loenen and W. Hofs-Kemper, Pharm. Weekbl., 1979, 1, 424-436.

26 H. Saito, M. Yokoi, M. Ishida and T. Asakura, Macromolecules, 1990, 23, 83-88.

27 H. Al-Sa'doni and A. Ferro, Clin. Sci., 2000, 98, 507-520.

28 A. Srinivasan, N. Kebede, J. E. Saavedra, A. V. Nikolaitchik, D. A. Brady, E. Yourd, K. M. Davies, L. K. Keefer and J. P. Toscano, J. Am. Chem. Soc., 2001, 123, 5465-5472.

29 E. T. Pashuck and S. I. Stupp, J. Am. Chem. Soc., 2010, 132, 8819-8821.

30 M. Hughes, H. Xu, P. W. J. M. Frederix, A. M. Smith, N. T. Hunt, T. Tuttle, I. A. Kinloch, R. V. Ulijn, L. Kale, K. Schulten, P. L. A. Popelier, M. L. Turner, P. Xiao, I. A. Kinloch and R. V. Ulijn, Soft Matter, 2011, 7, 10032.

31 D. M. Ryan, S. B. Anderson, F. T. Senguen, R. E. Youngman and B. L. Nilsson, Soft Matter, 2010, 6, 475-479.

32 B. Adhikari, J. Nanda and A. Banerjee, Soft Matter, 2011, 7, 8913.

33 S. Fleming, P. W. J. M. Frederix, I. Ramos Sasselli, N. T. Hunt, R. V. Ulijn and T. Tuttle, Langmuir, 2013, 29, 9510-9515.

34 S. Fleming, S. Debnath, P. W. J. M. Frederix, N. T. Hunt and R. V. Ulijn, Biomacromolecules, 2014, 15, 1171-1184.

35 D. B. Soria and P. J. Aymonino, Spectrochim. Acta, Part A, 1999, 55, 1243-1253.

36 P. K. Sukul and S. Malik, RSC Adv., 2013, 3, 1902-1915.
37 A. Baral, S. Basak, K. Basu, A. Dehsorkhi, I. W. Hamley and A. Banerjee, Soft Matter, 2015, 11, 4944-4951.

38 M. Cao, Y. Wang, X. Ge, C. Cao, J. Wang, H. Xu, D. Xia, X. Zhao and J. R. Lu, J. Phys. Chem. B, 2011, 115, 11862-11871.

39 M. R. Caplan, P. N. Moore, S. Zhang, R. D. Kamm and D. A. Lauffenburger, Biomacromolecules, 2000, 1, 627-631.

40 M. Nihei, Y. Yanai, I.-J. Hsu, Y. Sekine and H. Oshio, Angew. Chem., Int. Ed., 2017, 56, 591-594.

41 S. C. White and H. W. Thompson, Proc. R. Soc. London, Ser. A, 1966, 291, 460-468.

42 R. O. Ramabhadran, Y. Hua, A. H. Flood and K. Raghavachari, J. Phys. Chem. A, 2014, 118, 7418-7423.

43 G. Fichman, T. Guterman, L. Adler-abramovich and E. Gazit, CrystEngComm, 2015, 17, 8105-8112.

44 C. Berthomieu and R. Hienerwadel, Photosynth. Res., 2009, 101, 157-170.

45 W. Liyanage and B. L. Nilsson, Langmuir, 2016, 32, 787-799. 46 S. E. Wheeler, Acc. Chem. Res., 2013, 46, 1029-1038.

47 S. L. Cockroft, J. Perkins, C. Zonta, H. Adams, S. E. Spey, C. M. R. Low, J. G. Vinter, K. R. Lawson, C. J. Urch and C. A. Hunter, Org. Biomol. Chem., 2007, 5, 1062.

48 F. Cozzi, F. Ponzini, R. Annunziata, M. Cinquini and J. S. Siegel, Angew. Chem., Int. Ed. Engl., 1995, 34, 1019-1020. 49 N. Rubin, E. Perugia, S. G. Wolf, E. Klein, M. Fridkin and L. Addadi, J. Am. Chem. Soc., 2010, 132, 4242-4248.

50 W. Hoyer, T. Antony, D. Cherny, G. Heim, T. M. Jovin and V. Subramaniam, J. Mol. Biol., 2002, 322, 383-393.

51 L. R. Volpatti, M. Vendruscolo, C. M. Dobson and T. P. J. Knowles, ACS Nano, 2013, 7, 10443-10448.

52 P. A. Korevaar, C. J. Newcomb, E. W. Meijer and S. I. Stupp, J. Am. Chem. Soc., 2014, 136, 8540-8543.

53 F. Tantakitti, J. Boekhoven, X. Wang, R. V. Kazantsev, T. Yu, J. Li, E. Zhuang, R. Zandi, J. H. Ortony, C. J. Newcomb, L. C. Palmer, G. S. Shekhawat, M. O. de la Cruz, G. C. Schatz and S. I. Stupp, Nat. Mater., 2016, 15, 469-476.

54 E. C. Barker, A. D. Martin, C. J. Garvey, C. Y. Goh, F. Jones, M. Mocerino, B. W. Skelton, M. I. Ogden and T. Becker, Soft Matter, 2017, 13, 1006-1011.

55 Z. Yang, G. Liang, M. Ma, A. S. Abbah, W. W. Lu and B. Xu, Chem. Commun., 2007, 843-845.

56 Z. Yang, G. Liang, L. Wang and B. Xu, J. Am. Chem. Soc., 2006, 128, 3038-3043.

57 A. M. Smith, R. J. Williams, C. Tang, P. Coppo, R. F. Collins, M. L. Turner, A. Saiani and R. V. Ulijn, Adv. Mater., 2008, 20, 37-41.

58 M. Kabiri and L. D. Unsworth, Biomacromolecules, 2014, 15, 3463-3473.

59 J. Li, K. Fan, L. Niu, Y. Li and J. Song, J. Phys. Chem. B, 2013, 117, 5989-5995. 